\title{
Combinatorial Problems
}

\section{Foreword}

Combinatorial Mathematics, or "Combinatorics", regarded as originating in the Ars Combinatoria of Leibniz, has to do with problems of arrangement, operation, and selection within a finite or discrete system-such as the aggregate of all possible states of a digital computer. Until recently, preoccupation with continuous mathematics has inhibited the growth of discrete mathematics. But now it is realized that combinatorial methods can be developed to attack profitably, in modern science and technology, a vast variety of "problems of organized complexity" - an apt designation of Warren Weaver [1]. In 1947 Hermann Weyl [2] wrote as follows (rearranged slightly for quotation here):

\section{References}

1. Warren Weaver, "Science and Complexity", American Scientist, 36, 536 (1948).

2. Hermann Weyl, Philosophy of Mathematics and Natural Science, Princeton, 1949, p. 237.

3. John Riordan, An Introduction to Combinatorial Analysis, Wiley, 1958.

4. Marshall Hall, Jr., "A Survey of Combinatorial Analysis", Some Aspects of Analysis and Probability (Vol. IV of Surveys in Applied Mathematics), Wiley, 1958, p. 37.
"Perhaps the philosophically most relevant feature of modern science is the emergence of abstract symbolic structures as the hard core of objectivity behind - as Eddington puts it-the colorful tale of the subjective storyteller mind. The combinatorics of aggregates and complexes deals with some of the simplest such structures imaginable. It is gratifying that combinatorial mathematics is so closely related to the philosophically important problems of individuation and probability, and that it accounts for some of the most fundamental phenomena in inorganic and organic nature. This structural viewpoint occurs in the foundations of quantum mechanics. In a widely different field John von Neumann's and Oskar Morgenstern's attempt to found economics on a theory of games is characteristic of the same trend. The network of nerves joining the brain with the sense organs is a subject that by its very nature invites combinatorial investigation. Modern computing machines translate our insight into the combinatorial structure of mathematics into practice by mechanical and electronic devices."

Collected in this issue are papers concerned with an intriguing assortment of combinatorial problems and methods-centered on graphs (networks) and matrices, but ranging into combinatorial designs, codes, topology, programming and games. However, although the papers reach beyond the scope of classical Combinatorial Analysis [3], [4], they constitute only fragmentary anticipation of the broad and highly developed body of Combinatorial Mathematics that seems destined to evolve in the future.

These papers stem from a Research Institute on Combinatorial Problems held at the IBM Research Center, Yorktown Heights, N. Y., July 6 to August 14,1959 . Some of the papers were also presented at a Symposium on Combinatorial Problems, sponsored by the IBM Corporation and the Society for Industrial and Applied Mathematics (SIAM), at Princeton University, April 11-13, 1960. Herman Goldstine, James Griesmer and Paul Roth of the IBM Research Center played a leading part in promoting these activities. In particular, it was Paul Roth who (with the undersigned) initiated the chain of events that culminates in this special issue of the IBM Journal.

\section{A. W. Tucker}

